\title{
An Electrode Configuration for Recording Muscle Motor Evoked Potentials in the Upper Extremities during Intraoperative Neurophysiological Monitoring
}

\author{
Young-Doo Choi, B.S., ${ }^{1}$ Seung-Hyun Jin, Ph.D., ${ }^{1,2}$ Chi-Heon Kim, M.D., Ph.D., ${ }^{1,2,3}$ Gil Ho Kwak, B.S., Bo Eun Kim, B.S., \\ Chun Kee Chung, M.D., Ph.D. ${ }^{1,2,3,4}$ \\ Department of Neurosurgery,' Seoul National University Hospital, Seoul National University College of Medicine, Seoul, Korea \\ Neuroscience Research Institute, ${ }^{2}$ Seoul National University Medical Research Center, Seoul, Korea \\ Department of Neurosurgery, ${ }^{3}$ Seoul National University College of Medicine, Seoul, Korea \\ Department of Brain and Cognitive Sciences, ${ }^{4}$ Seoul National University College of Natural Sciences, Seoul, Korea
}

Objective : The main aim of the present study is to examine the electrode configurations used to record the muscle motor evoked potential (MMEP) in the upper extremities during surgery with the goal of producing a high and stable mMEP signal, in particular among the abductor pollicis brevis (APB), abductor digiti minimi (ADM), and across the APB-ADM muscles, which have been widely used for the MMEP in the upper extremities.

Methods : Thirty right-handed patients were recruited in this prospective study. No patients showed any adverse events in their mMEP signals of the upper extremities during surgery. The mMEPs were recorded independently from the signals for the APB and ADM and for those across the APB-ADM.

Results : The mMEP amplitude from across the APB-ADM was statistically higher than those recorded from the APB and ADM muscles. Moreover, the coefficient of variation of the mMEP amplitude from across the APB-ADM was smaller than those of mMEP amplitude recorded from the APB and ADM muscles.

Conclusion : The mMEP from across the APB-ADM muscles showed a high yield with high stability compared to those in each case from the APB and ADM muscles. The configuration across the APB-ADM muscles would be best for mMEP recordings from the upper extremities for intraoperative neurophysiological monitoring purposes.

Key Words : Motor evoked potential · Intraoperative neurophysiological monitoring $\cdot$ Upper extremity $\cdot$ Abductor digiti minimi . Abductor pollicis brevis · Across APB-ADM.

- Received : June 2, 2016 •Revised : December 31, 2016 •Accepted : January 5, 2017

- Address for reprints : Chun Kee Chung, M.D., Ph.D.

Department of Neurosurgery, Seoul National University Hospital, Seoul National University College of Medicine, 101 Daehak-ro, Jongno-gu, Seoul 03080, Korea Tel : +82-2-2072-2352, Fax : +82-2-744-8459, E-mail : chungc@snu.ac.kr

This is an Open Access article distributed under the terms of the Creative Commons Attribution Non-Commercial License (http://creativecommons.org/licenses/by-nc/4.0) which permits unrestricted non-commercial use, distribution, and reproduction in any medium, provided the original work is properly cited. 


\section{INTRODUCTION}

Intraoperative neurophysiological monitoring (INM) has received a great deal of attention as a means of protecting patients from neurologic injuries during surgery ${ }^{6}$. Among the various INM techniques developed thus far, muscle motor evoked potential (mMEP) monitoring allows an ongoing assessment of the functional integrity of descending motor tracts during surgery when special attention regarding motor functions is needed ${ }^{1)}$. The potentials evoked by transcranial electric stimulation can be recorded from the limb muscles, known as the muscle mMEPs. The appropriate muscles for monitoring the lower extremities are usually the tibialis anterior (TA) and the abductor hallucis ( $\mathrm{AH}$ ) brevis ${ }^{3)}$. However, different sensitivity and clinical significance levels between the TA and AH muscles have been reported ${ }^{4)}$. The preferred muscles for monitoring the mMEPs in the upper extremities are the abductor digiti minimi (ADM), first dorsal interosseous, abductor pollicis brevis (APB) as well as the flexors and extensors of the forearm ${ }^{2}$. Although different muscles of the extremities can monitor different peripheral nerves and nerve roots ${ }^{5)}$, mMEPs generally exhibit variability in the amplitude, threshold, and morphology, attributed to the fluctuating excitability of the lower motor neuron pool ${ }^{5)}$. In addition, the degree of inconsistency between patients and muscles makes it difficult to define specific warning criteria $^{5)}$. Therefore, mMEP recordings with both a high yield and stability at the same time would be beneficial for monitoring during surgery.

\section{MATERIALS AND METHODS}

\section{Subjects}

A total of 30 right-handed patients (mean \pm standard deviation : $25.95 \pm 3.85$ years, ranging from 18 to 77,24 males) were enrolled in this prospective study (Table 1).

Patients who underwent brain or spine surgery with the use of INM between May of 2013 and July of 2013 at Seoul National University Hospital were included. Patients who had motor dysfunction, numbness and tumors adjacent to the motor cortex were excluded. Two of the 30 patients who showed the event of a mMEP change during surgery were excluded from the analysis. Because, finding of this study is reference values of MEP monitoring technique at APB, ADM, and across APB-ADM in INM. Further studies are required to demonstrate the accuracy of this technique with clinical outcome and to establish its use in follow-up trials. The types of surgery include removal of a brain tumor $(n=16)$, superficial temporal artery to middle cerebral artery anastomosis $(n=13)$, and endoscopic discectomy of the lumbar spine $(n=1)$.

There is one patient of disc disease of lumbar spine who was monitored both upper extremities like thenar and both lower extremities.

The reason why monitoring upper extremity in lumbar spine surgery, is to compare reference value of upper extremity and lower extremity. Sometimes MEP changes were occurred during operation, and there are many reasons of evoked potential change, influence of anesthesia or systemic factors, patient positioning problem. At that situation, upper extremity monitoring can provide the reason more exactly.

The study protocol was approved by the local Institutional Review Board at Seoul National University (IRB No.: H-1301102-458).

\section{The protocol of intraoperative monitoring}

General anesthesia was induced with propofol $(2 \mathrm{mg} / \mathrm{kg})$ and remifentanyl $(1 \mu \mathrm{g} / \mathrm{kg})$. A muscle relaxant was given once before intubation and was not given thereafter. Intraoperatively, propofol was maintained at $2-10 \mathrm{mg} / \mathrm{kg} / \mathrm{hr}$ and remifentanyl at $15 \mu \mathrm{g} / \mathrm{kg} / \mathrm{hr}$. The mean blood pressure remained higher than $90 \mathrm{mmHg}$.

Transcranial anodal motor cortex stimulation was performed with electrical pulses through sterile needle electrodes inserted over motor cortex regions at C3' and C4' corresponding to $1 \mathrm{~cm}$ posterior to C3 and C4 (international 10-20 system). Trains of five pulses (biphasic square) with an interstimuli interval of $1-2 \mathrm{~ms}$ at a rate of $1 \mathrm{~Hz}$ were delivered by a commercially available transcortical stimulator (Eclipse, Axon Systems Inc., New York, NY, USA). The stimulation intensity level was $300 \mathrm{~V}$. However, at a low mMEP amplitude, the stimulation intensity was gradually increased $(\sim 500 \mathrm{~V})$ until stable MEPs of at least $200 \mu \mathrm{V}$ were obtainable in all target muscles. Signals were filtered between 30 and $1000 \mathrm{~Hz}$.

In the upper extremities, MEPs were recorded from the bilateral deltoid muscles and from three locations in the hand. The three recording locations were the APB, ADM, and across the APB-ADM. For the APB and ADM muscles, both active and reference electrodes were placed on each muscle; more- 
Intraoperative mMEP in the Upper Extremities | Choi YD, et al.

Table 1. Diagnoses and mMEP amplitude and latency recorded from APB, ADM and across APB-ADM

\begin{tabular}{|c|c|c|c|c|c|c|c|c|c|c|}
\hline \multirow[b]{2}{*}{ No. } & \multirow[b]{2}{*}{ Age } & \multirow[b]{2}{*}{ Sex } & \multirow[b]{2}{*}{ Diagnosis } & \multirow{2}{*}{$\begin{array}{c}\text { OP } \\
\text { event }\end{array}$} & \multicolumn{2}{|c|}{ APB } & \multicolumn{2}{|c|}{ ADM } & \multicolumn{2}{|c|}{ Across APB-ADM } \\
\hline & & & & & $\begin{array}{l}\text { Amplitude } \\
(\mu \mathrm{v})\end{array}$ & $\begin{array}{c}\text { Latency } \\
\text { (msec) }\end{array}$ & $\begin{array}{l}\text { Amplitude } \\
(\mu \mathrm{v})\end{array}$ & $\begin{array}{c}\text { Latency } \\
\text { (msec) }\end{array}$ & $\begin{array}{l}\text { Amplitude } \\
(\mu \mathrm{v})\end{array}$ & $\begin{array}{l}\text { Latency } \\
\text { (msec) }\end{array}$ \\
\hline 1 & 23 & $M$ & Moyamoya & + & $N / A$ & $N / A$ & $N / A$ & $N / A$ & $N / A$ & $N / A$ \\
\hline 2 & 70 & M & Glioma & - & 1020.2 & 26.78 & 2846.2 & 25.68 & 2294.8 & 24.52 \\
\hline 3 & 18 & M & Glioma & - & 670.8 & 20.6 & 161.8 & 24.42 & 780.6 & 20.92 \\
\hline 4 & 37 & M & Disc disease of lumbar & - & 2697.8 & 26.16 & 1163.6 & 26.12 & 2750.2 & 22.78 \\
\hline 5 & 44 & M & Moyamoya & + & N/A & $N / A$ & $N / A$ & N/A & N/A & N/A \\
\hline 6 & 34 & M & Brain tumor & - & 1664.6 & 24.38 & 850.8 & 26.88 & 1524 & 24.1 \\
\hline 7 & 55 & M & Glioma & - & 2022.2 & 23.58 & 1317.2 & 24.24 & 2453.6 & 22.36 \\
\hline 8 & 65 & M & Glioma & - & 4011 & 20.84 & 1008.8 & 21.14 & 5586.4 & 21.7 \\
\hline 9 & 54 & M & Glioma & - & 2311.4 & 24.7 & 583 & 22.48 & 2641.8 & 23.2 \\
\hline 10 & 37 & $M$ & $\begin{array}{l}\text { Cerebral arteriovenous } \\
\text { malformation }\end{array}$ & - & 2533.4 & 21.32 & 819 & 22.14 & 2959.8 & 20.42 \\
\hline 11 & 58 & $M$ & Aneurysm & & 2007.6 & 23.66 & 904.2 & 20.02 & 1453.4 & 19.78 \\
\hline 12 & 51 & $\mathrm{~F}$ & Moyamoya & & 2111.2 & 20.44 & 1119.8 & 23.14 & 3231 & 20.28 \\
\hline 13 & 40 & $\mathrm{~F}$ & Glioma & - & 617.8 & 20.86 & 87.68 & 25.46 & 396.8 & 20.44 \\
\hline 14 & 62 & M & $\begin{array}{l}\text { Internal carotid artery } \\
\text { occlusion }\end{array}$ & - & 2151.2 & 25.2 & 1216.4 & 23.42 & 1467.6 & 23.08 \\
\hline 15 & 66 & M & Glioblastoma & - & 1042.2 & 22.2 & 853.4 & 22.58 & 1643 & 22.74 \\
\hline 16 & 42 & $\mathrm{~F}$ & Moyamoya & - & 400.8 & 20.12 & 882.8 & 19.94 & 1087.2 & 20.06 \\
\hline 17 & 23 & $\mathrm{~F}$ & Moyamoya & - & 402.2 & 20.28 & 33.42 & 21.18 & 473.4 & 20.4 \\
\hline 18 & 54 & M & Glioma & - & 310.4 & 22.32 & 85.3 & 25.32 & 422.2 & 23.98 \\
\hline 19 & 57 & M & Aneurysm & - & 2290.8 & 21.8 & 1036.6 & 21.32 & 4645 & 21.92 \\
\hline 20 & 77 & M & Brain tumor & - & 2233 & 22.28 & 838.8 & 22.18 & 4197.6 & 22.44 \\
\hline 21 & 61 & M & Glioma & - & 1281 & 22.56 & 1137.4 & 22.8 & 3853.2 & 22.7 \\
\hline 22 & 44 & M & Aneurysm & - & 1266.6 & 20.74 & 77.8 & 20.04 & 2359.2 & 20.3 \\
\hline 23 & 51 & $\mathrm{~F}$ & Moyamoya & - & 3089.8 & 19.54 & 1444.4 & 19.42 & 8349.6 & 19.32 \\
\hline 24 & 25 & M & Glioma & - & 291.2 & 25.54 & 30.08 & 26.12 & 421 & 23.1 \\
\hline 25 & 73 & M & Glioblastoma & - & 2124.6 & 22.98 & 1393.2 & 24.74 & 2114.8 & 24.72 \\
\hline 26 & 53 & M & Cerebral infarction, MCA & - & 1167.6 & 21.52 & 578.2 & 22.96 & 3695.2 & 22.54 \\
\hline 27 & 77 & M & Glioma & - & 1385.8 & 23.92 & 1057.2 & 24.62 & 1085.6 & 24.2 \\
\hline 28 & 30 & $\mathrm{~F}$ & Tuberous sclerosis & - & 2282 & 20.76 & 245.8 & 20.2 & 1784 & 21.02 \\
\hline 29 & 49 & M & Aneurysm & - & 2793.4 & 22.1 & 686.6 & 23.16 & 4225.2 & 22.92 \\
\hline 30 & 61 & M & Meningioma & - & 3533.6 & 22.8 & 1088 & 24.66 & 2476.8 & 22.32 \\
\hline \multicolumn{4}{|c|}{ Total mean value } & & 1775.5 & 22.5 & 841 & 23.08 & 2513.3 & 22.08 \\
\hline
\end{tabular}

mMEP : muscle motor evoked potential, APB : abductor pollicis brevis, ADM : abductor digiti minimi, OP : operation, $\mathrm{M}:$ male, + : present, N/A : not available, - : absent, F : female, MCA : middle cerebral artery

over, two electrodes, an active electrode on the APB and a reference on the ADM, were placed to record the typical hand muscle reactivity to the motor cortex stimulation, referred to here as across the APB-ADM.
Neurophysiological monitoring was done throughout the surgery. The baseline mMEP was obtained after anesthetic induction and before the major procedure. For the mMEP, the peak-to-peak amplitude and latency were continuously moni- 


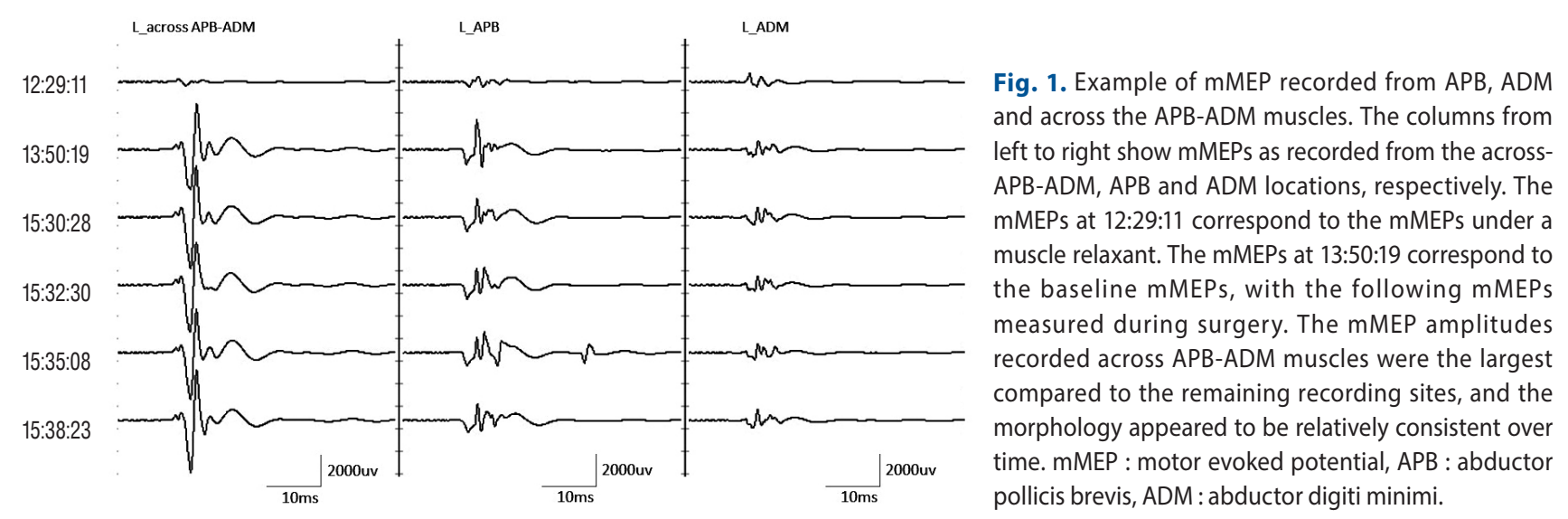

tored through the operation. MEPs were checked approximately every 10 minutes, and additional stimulation was applied upon the surgeon's request

\section{Statistical analysis}

The Kolmogorov-Smirnov test was performed to test the distribution normality of the data. Differences in mMEP amplitudes and the related coefficient of variation $(\mathrm{CV})$ values among the mMEP detection sites (i.e., APB, ADM, and across the APB-ADM) were evaluated with nonparametric statistics, i.e., the Friedman test. A post-hoc analysis with the Wilcoxon signed-rank test was conducted with Bonferroni correction applied, resulting in a significance level of $p<0.05$. All analyses were done with the Statistical Package for the Social Sciences, version 15.0 for Windows (SPSS Inc., Chicago, IL, USA).

\section{RESULTS}

\section{The results of the 28 operations performed (Table 1)}

Fig. 1 shows the mMEP amplitude recorded from the APB, ADM and across the APB-ADM muscles. The mMEP peakto-peak amplitudes differ depending on the recording site. Through a visual inspection, the mMEP amplitudes recorded across the APB-ADM muscles were the largest compared to the remaining recording sites, and the morphology remained relatively consistent over time.

Fig. 2 shows the statistical differences in the mMEP amplitudes among the different recording methods. A comparison of the repeated measures was conducted using Friedman's test, showing statistically significant differences between the

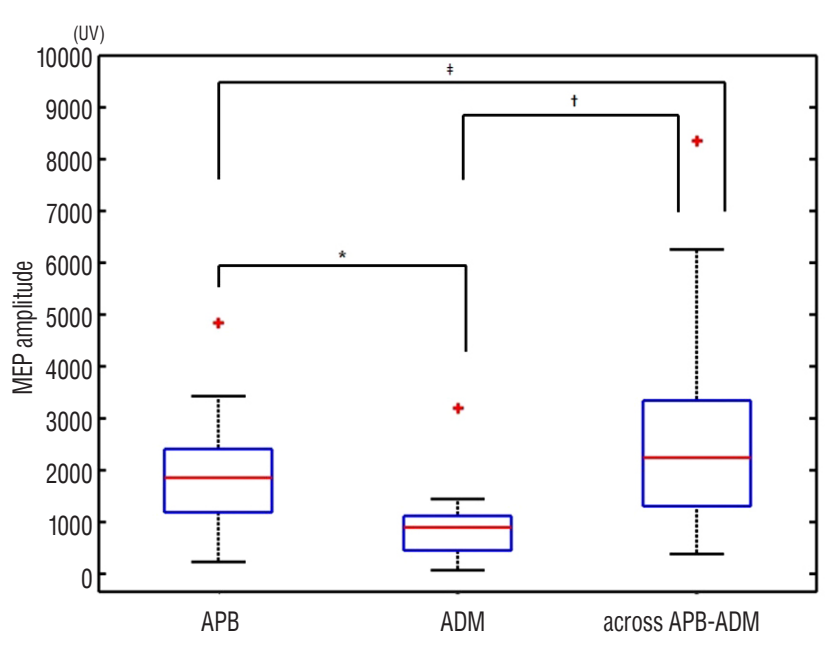

Fig. 2. Boxplot of the mMEP amplitude recorded from the APB, ADM and across the APB-ADM muscles. The $y$-axis indicates the mMEP amplitude (unit: $\mu V$ ). Median mMEP amplitude values recorded from the APB, ADM and across the APB-ADM were $1853.9 \mu \mathrm{V}, 893.0 \mu \mathrm{V}, 2238.0 \mu \mathrm{V}$, respectively. Wilcoxon signed-rank tests with Bonferroni correction showed significant differences between APB and ADM $\left({ }^{*} p<0.01, r=0.74\right)$ and between the ADM and across-APB-ADM values ( ${ }^{\dagger} p<0.01, r=0.83$ ). But between the APB and across-APB-ADM values, there were not showed significant differences $\left({ }^{\ddagger} p<0.05, r=0.49\right)$. mMEP : motor evoked potential, APB : abductor pollicis brevis, ADM : abductor digiti minimi.

mMEP amplitudes taken with the different recording methods $\left(\chi^{2}(2)=36.50, p<0.001\right)$. A post-hoc test using Wilcoxon signed-rank tests with Bonferroni correction showed significant differences between APB and ADM $(p<0.01, \mathrm{r}=0.74)$ and between ADM and across the APB-ADM $(p<0.01, r=0.83)$. But between APB and across the APB-ADM, there were not showed significant differences $(p<0.05, \mathrm{r}=0.49)$. The mMEP amplitude recorded from across the APB-ADM muscles presented the largest amplitude compared to those recorded from the APB and ADM muscles. 


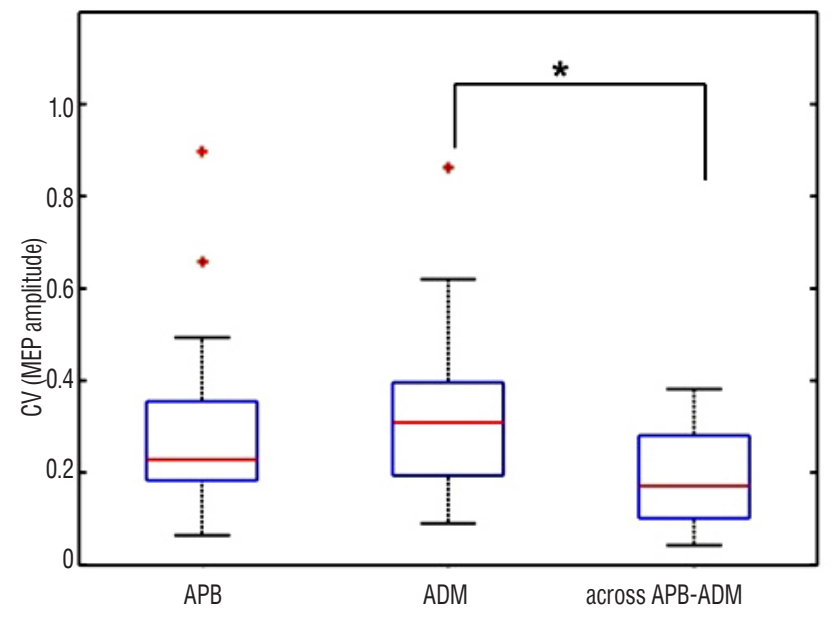

Fig. 3. Boxplot of the coefficient of variation (CV) of the mMEP amplitudes recorded from the APB and ADM and from across the APBADM muscles. The median CV of recorded from the APB, ADM and across-APB-ADM locations were $0.23,0.31$, and 0.17 , respectively. Wilcoxon signed-rank tests with Bonferroni correction showed a larger amplitude recorded from across the APB-ADM muscles compared to that recorded from $A D M\left({ }^{*} p<0.01, r=0.65\right)$. No significant differences were detected between APB and ADM and between APB and across the APBADM. mMEP : motor evoked potential, APB : abductor pollicis brevis, ADM : abductor digiti minimi.

Fig. 3 shows the $\mathrm{CV}$ of the mMEP amplitude recorded from the APB, ADM and across the APB-ADM muscles. A comparison of the repeated measures was performed using Friedman's test, showing statistically significant differences of the $\mathrm{CV}$ of the mMEP amplitudes among the recording methods $\left(\chi^{2}(2)=9.50, p<0.01\right)$. A post-hoc test using Wilcoxon signedrank tests with Bonferroni correction showed significant differences in the ADM and across the APB-ADM results, demonstrating a larger amplitude recorded from across the APB$\mathrm{ADM}$ muscles than that recorded from $\mathrm{ADM}(p<0.01, \mathrm{r}=0.65)$. However, no significant differences were detected between $\mathrm{APB}$ and $\mathrm{ADM}(p=0.43, \mathrm{r}=0.15)$ or between APB and across the APB-ADM muscles $(p=0.02, \mathrm{r}=0.43)$.

\section{DISCUSSION}

In neurosurgery operation, there are many EMG were awaked through operating nearby peripheral nerve. Therefore the situation of patient's neuronal motor function through INM were needed by simultaneously during operation. So there were needed more precisely monitoring technic to INM. The main aim of the present study was to determine which electrode configuration when recording mMEPs in the upper extremities would produce high and stable mMEPs among APB, ADM, and across the APB-ADM muscles, all of which are widely used muscles for mMEP monitoring in the upper extremities during surgery. The present study showed that the mMEP amplitude recorded from across the APB-ADM muscles was statistically high compared to those recorded from the ADM muscles.

However, no significant differences were detected between APB and ADM $(p=0.43, r=0.15)$ and between the APB and across the APB-ADM results ( $p=0.02, r=0.43$ ) at $C V$ value.

Given that the degree of inconsistency between patients and muscles makes it difficult to define specific warning criteria ${ }^{5}$, a mMEP recording method with both a high yield and stability simultaneously would be beneficial to those who monitor the functional integrity of corticospinal tracts during surgery.

The across-muscle MEP values were higher than the singlemuscle MEP value because two muscles were moved independently by each nerve at some distance from each other. Amplitude is the objective measurement of the degree of change from positive to negative. The muscle across the APB-ADM has some distance from positive to negative. The APB muscle moved when the median nerve was stimulated, and the ADM muscle moved when the ulnar nerve was stimulated. Recording MEPs from across two muscles can reflect the movements of the two muscles. The evoked potentials from two muscles were higher than those from one muscle. Because each muscle was moved independently during MEP stimulation from the motor cortex, this difference creates a higher MEP amplitude value compared to the MEP amplitude value of a single muscle.

This across-recording MEP also reflects the action potential of compound muscles. From this basis, our results suggest that the electrode configuration across the APB-ADM muscles would be the best $\mathrm{mMEP}$ recording configuration for the upper extremities, as the mMEP amplitude recorded from across the APB-ADM muscles was high and relatively stable compared to those recorded from the APB and ADM muscles.

The use of mMEPs for the purpose of the ongoing assessment of the functional integrity of corticospinal motor tracts during surgery is common ${ }^{1,6)}$. Several factors, such as the surgical sites, levels and strategies when determining the muscles and their recording configurations should be monitored during surgery. Although the TA and AH muscles are the typical muscles used for mMEP monitoring in the lower extremities, 
mMEPs are generated differently in the TA and AH muscles, which may influence the efficacy of the mMEP process ${ }^{4)}$. For the upper extremities, the ADM, APB, across-APB-ADM muscles have been used for mMEP monitoring during surgery, but the mMEP differences among different monitoring configurations have never been investigated. We showed for the first time that the electrode configuration across the APB-ADM muscles would be the best $\mathrm{mMEP}$ recording configuration for the upper extremities in terms of amplitude and stability.

The generation of mMEPs during anesthesia depends on the excitability of the alpha-motoneurones in the anterior horns. In this case, mMEPs can be generated only if the resting potential of the alpha-motoneurones reaches the firing threshold and thus transmits this activity via the motor axons of the peripheral nerves and the neuromuscular junctions to the muscle ${ }^{2}$. Therefore, the generation of mMEPs can be indicative of the functional integrity of the corticospinal motor tracts, which is the basis of the use of mMEPs for INM purposes. However, one concern is that mMEPs generally vary in terms of their amplitude, threshold, and morphology $y^{5)}$, and the degree of inconsistency between patients and muscles makes it difficult to define specific warning criteria ${ }^{5}$. Accordingly, as hypothesize that mMEP recordings with both a high yield and good stability at the same time would be beneficial for monitoring purposes during surgery. In addition, it has been reported that the grip strength during motor recovery after a subcortical stroke is correlated with the mMEP amplitude, suggesting that the grip strength depends on the restoration of corticospinal excitability ${ }^{7)}$. But This study finding did not implies a high yield of mMEP recording from across the APB-ADM muscles would be a good indicator of hand function after surgery. Clinically futher study needs to reflect that this method can indicate the hand motor function.

One limitation of the present study is that because two patients who showed the event of a mMEP change during surgery were excluded from the analysis, we were unable to evaluate the prognostic usefulness of using mMEP in the upper extremities depending on the recording configuration. However, the main aim of the study was to compare the yield and its stability of mMEP when recorded from the APB and ADM and from across the APB-ADM muscles on the basis of a high yield and stability at the same time. The evaluation of the prognostic values of mMEP in the upper extremities depending on the recording configuration is beyond the scope of the present study.

\section{CONCLUSION}

In conclusion, compared to those recorded from the APB and ADM muscles, our findings revealed that mMEPs recorded from across the APB-ADM muscles demonstrated a high yield with high stability, implying that the electrode configuration across the APB-ADM muscles would be an adequate mMEP recording configuration on the upper extremities considering INM purposes.

\section{- Acknowledgements}

This research was supported by the Brain Research Program through the National Research Foundation of Korea (NRF) funded by the Ministry of Science, ICT \& Future Planning (2016M3C7A1904984).

This research was supported by Basic Science Research Program through the National Research Foundation of Korea (NRF) funded by the Ministry of Education (No. 2015R1D1A1A 02061486).

\section{References}

1. Anil Mendiratta, Emerson RG : Transcranial electrical mMEP with muscle recording, in Nuwer MR (ed) : Intraoperative Monitoring of Neural Function. Amsterdam : ELSEVIER, 2008, pp260-272

2. Deletis $V$, Sala F : Corticospinal tract monitoring with D- and I-waves from the spinal cord and muscle MEPs from limb muscles, in Nuwer MR (ed) : Intraoperative Monitoring of Neural Function. Amsterdam : ELSEVIER, 2008, pp235-251

3. Jankowska E, Padel Y, Tanaka R : Projections of pyramidal tract cells to alpha-motoneurones innervating hind-limb muscles in the monkey. J Physiol 249 : 637-667, 1975

4. Kim SM, Yang H, Park SB, Han SG, Park KW, Yoon SH, et al. : Patternspecific changes and discordant prognostic values of individual leg-muscle motor evoked potentials during spinal surgery. Clin Neurophysiol 123 : 1465-1470, 2012

5. Macdonald DB, Stigsby B, Al Homoud I, Abalkhail T, Mokeem A : Utility of motor evoked potentials for intraoperative nerve root monitoring. J Clin Neurophysiol 29 : 118-125, 2012

6. Nuwer MR: Overview and history, in Nuwer MR (ed) : Intraoperative Monitoring of Neural Function. Amsterdam : ELSEVIER, 2008, pp2-6

7. Thickbroom GW, Byrnes ML, Archer SA, Mastaglia FL : Motor outcome after subcortical stroke: MEPs correlate with hand strength but not dexterity. Clin Neurophysiol 113 : 2025-2029, 2002 